\title{
Laminar Motion of the Incompressible Fluids in Self-Acting Thrust Bearings with Spiral Grooves
}

\author{
Cornel Velescu ${ }^{1}$ and Nicolae Calin Popa ${ }^{2}$ \\ ${ }^{1}$ Hydraulic Machinery Department, Mechanical Faculty, "Politehnica" University of Timisoara, Boulevard Mihai Viteazul No. 1, \\ 300222 Timisoara, Romania \\ ${ }^{2}$ Center for Advanced and Fundamental Technical Research (CAFTR), Romanian Academy-Timisoara Branch, \\ Boulevard Mihai Viteazul No. 24, 300223 Timisoara, Romania \\ Correspondence should be addressed to Nicolae Calin Popa; ncpopa@flumag2.mec.upt.ro
}

Received 4 October 2013; Accepted 28 November 2013; Published 2 January 2014

Academic Editors: M. Kuciej, F. Liu, and L. Nobile

Copyright (C) 2014 C. Velescu and N. C. Popa. This is an open access article distributed under the Creative Commons Attribution License, which permits unrestricted use, distribution, and reproduction in any medium, provided the original work is properly cited.

\begin{abstract}
We analyze the laminar motion of incompressible fluids in self-acting thrust bearings with spiral grooves with inner or external pumping. The purpose of the study is to find some mathematical relations useful to approach the theoretical functionality of these bearings having magnetic controllable fluids as incompressible fluids, in the presence of a controllable magnetic field. This theoretical study approaches the permanent motion regime. To validate the theoretical results, we compare them to some experimental results presented in previous papers. The laminar motion of incompressible fluids in bearings is described by the fundamental equations of fluid dynamics. We developed and particularized these equations by taking into consideration the geometrical and functional characteristics of these hydrodynamic bearings. Through the integration of the differential equation, we determined the pressure and speed distributions in bearings with length in the "pumping" direction. These pressure and speed distributions offer important information, both quantitative (concerning the bearing performances) and qualitative (evidence of the viscous-inertial effects, the fluid compressibility, etc.), for the laminar and permanent motion regime.
\end{abstract}

\section{Introduction}

Incompressible fluid motion in "self-acting thrust bearings with spiral grooves" (SATBESPIG) is a complex motion in thin layers [1-5] bounded by two solid surfaces in relative rotation one to another. The incompressible fluid motion in thin layers has as mathematical consequence the simplification and particularization of the general equations of motion. We made this simplification of the general equations of motion by neglecting the terms smaller by more than one order of magnitude [1-7] than the other terms. Starting from this observation, the paper approaches the theoretical study of incompressible fluid motion in SATBESPIG with inner and exterior pumping, working in the laminar regime.

Some authors have developed comparative theoretical and experimental studies concerning different aspects of spiral groove bearings functioning in the laminar regime, with equations for their functioning using magnetic fluids, cavitation phenomenon, and the geometry of the bearings with spiral/axial multiples grooves.

In $[8,9]$, the author studied the static and dynamic performances of the bearings with spiral grooves and inner and exterior pumping, working with compressed air. A comparative study of the theoretical and experimental results was presented.

The diversification and optimization of the geometry of the bearings with spiral or axial multiples of grooves were approached in [10-12]. In [13], the author analyzed the cavitation phenomenon theoretically and experimentally in bearings with spiral grooves working in mineral oil. The unfavorable consequences of the cavitation were evaluated.

We consider the theoretical results as a first step in our intention to approach the functionality of the hydrodynamic bearings in general and of the thrust bearings with spiral grooves in particular in the stationary/nonstationary turbulent regime. The viscous compressible/incompressible fluid 


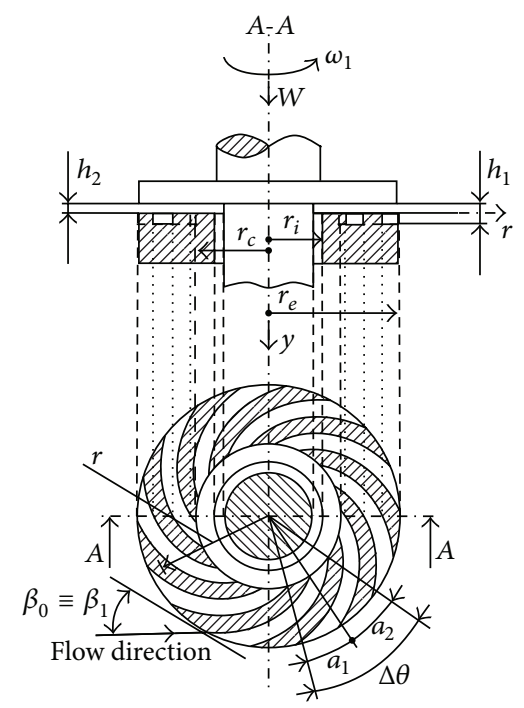

FIGURE 1: SATBESPIG and inner pumping.

motion can be approached by taking into consideration the thermo-viscous-inertial phenomena ensemble that exists in bearings.

\section{Constructive Geometric and Cinematic Elements of the SATBESPIG}

The SATBESPIG (Figures 1 and 2) are special hydrodynamic bearings that have the specific effect of "autopumping" $[1-3,5$, 7], which is why we are interested in the incompressible fluid motion in the laminar and permanent regime and, taking into consideration the influence of the inertial forces, in the length of the spiral groove (pumping direction $\psi$ from Figures 3, 4, and 5). In the literature $[4,7,10,12-15]$, there are only a few studies and a small amount of theoretical research concerning this subject. These studies approach the incompressible fluid motion only in the radial direction $(r)$ without taking into consideration the effects of the inertial forces.

In Figures 1 and 2, (i) $a_{1}$ is the width of the spiral channel, measured on the circle arc contour; (ii) $a_{2}$ is the width of the spiral threshold, measured on the circle arc contour; (iii) $\Delta \theta$ is the center angle corresponding to a channel-threshold pair; (iv) $\beta_{0}$ is the generator angle of the logarithmic spiral, which describes the form of the cannels; (v) $\beta_{1}$ and $\beta_{2}$ are the input and output angles, respectively, in and from the channel (according to the accepted notations from the general study of the hydraulic machineries); (vi) $\omega_{1}$ is the bearing angular rotation speed; (vii) $r_{i}$ and $r_{e}$ are the inner and external radius of the bearing, respectively; (viii) $r_{c}$ is the radius marking the zone of the spiral channels; (ix) $r$ is the current radius of the bearing; (x) $h_{1}$ is the lubrication film height over the bearing channels; (xi) $h_{2}$ is the lubrication film height over the bearing thresholds; (xii) $W$ is the bearing load (the weight); and (xiii) by definition, $\alpha=a_{1} /\left(a_{1}+a_{2}\right)$.

With the geometrical dimensions in Figure 1 (or Figure 2) we can write $\Delta \theta=2 \pi / n_{p}$, where $n_{p}$ is the number of pairs

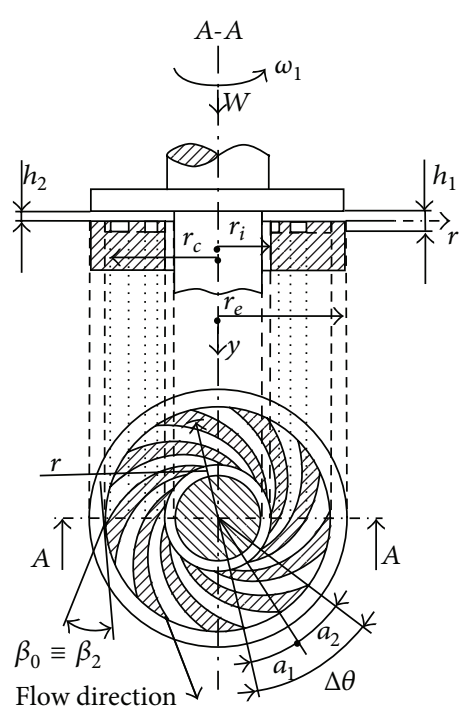

FIGURE 2: SATBESPIG and external pumping.

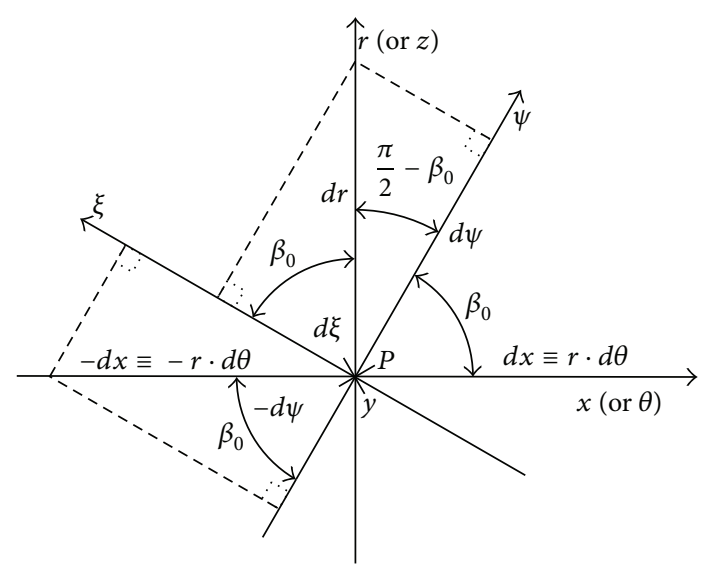

FIGURE 3: Relations between the general and local coordinate systems (in an arbitrary point, $P$ ). At point $P$, the coordinate $\xi$ is normal to the $\psi$ direction of the spiral channel.

channel-thresholds, $a_{1}=\alpha r \Delta \theta, a_{2}=(1-\alpha) r \Delta \theta, a=a_{1}+$ $a_{2}=r \Delta \theta$, and $\omega_{2}=0$ (the grooved surface is fixed).

\section{Coordinate Systems, Control Volume, Speed Distributions, and Mass Flow Rate in Laminar Regime}

To study the mathematical model, the coordinate systems and the control volume must be determined so as to define the speed distributions and the fluid mass flow rate $[3,5$, 15]. The control volume ( $\mathrm{Vol})$ is the volume between the bearing surfaces, between the one channel surface and one consecutive threshold surface of the stator and the horizontal bottom surface of the rotor (Figures 1 and 2). In Figure 3, we show the general $(y, r, \theta)$ and local $(\psi, y, \xi)$ coordinate systems used for the motion study. 
Next, we start from the fact that the incompressible fluid laminar motion, between the two quasiparallel surfaces of the bearing, is described by the following speed profiles:

$$
\begin{gathered}
u(y) \cong-\frac{1}{2 \eta} \frac{1}{r} \frac{\partial p}{\partial \theta} y(h-y)+\frac{y}{h} \omega_{1} r, \\
v_{n}(y) \cong 0, \\
w(y) \cong-\frac{1}{2 \eta} \frac{\partial p}{\partial r} y(h-y), \\
v_{\psi}(y) \cong \frac{1}{2 \eta r \sin \beta_{0}} \frac{\partial p}{\partial \psi} y(y-h)+\frac{y}{h} \omega_{1} r \cos \beta_{0}, \\
v_{\xi}(y) \cong \frac{1}{2 \eta r \sin \beta_{0}} \frac{\partial p}{\partial \xi} y(y-h)-\frac{y}{h} \omega_{1} r \sin \beta_{0} .
\end{gathered}
$$

In (1a)-(1e), (i) $u(y)$ is the fluid speed component in the $x$ (or $\theta$ ) direction, (ii) $v_{n}(y)$ is the fluid speed component in the normal direction $y$, (iii) $w(y)$ is the fluid speed component in the $z$ (or $r$ ) direction, (iv) $v_{\psi}(y)$ is the fluid speed component in the $\psi$ curb direction, (v) $v_{\xi}(y)$ is the fluid speed component in the $\xi$ direction, (vi) $p$ is the pressure in the fluid, (vii) $h$ is the height of the lubrication film, and (viii) $\eta$ is the fluid dynamical viscosity.

The speed distributions, given by (la)-(le), are typical for the noninertial motion case initially considered, meaning that these are parabolic speed profiles $[3,5,6]$. Observing the geometry of the bearings in Figures 1 and 2, it is possible to establish some functional mathematical relations between the coordinates. Thus, the following operational expressions can be found $[3,5]$ :

$$
\begin{gathered}
\frac{\partial}{\partial r}=\cos \beta_{0} \frac{\partial}{\partial \xi}+\sin \beta_{0} \frac{\partial}{\partial \psi}, \\
\frac{\partial}{\partial x} \equiv \frac{1}{r} \frac{\partial}{\partial \theta}=-\sin \beta_{0} \frac{\partial}{\partial \xi}+\cos \beta_{0} \frac{\partial}{\partial \psi} \\
\frac{\partial}{\partial \xi}=\cos \beta_{0} \frac{\partial}{\partial r}-\sin \beta_{0} \frac{1}{r} \frac{\partial}{\partial \theta}, \\
\frac{\partial}{\partial \psi}=\sin \beta_{0} \frac{\partial}{\partial r}+\cos \beta_{0} \frac{1}{r} \frac{\partial}{\partial \theta} .
\end{gathered}
$$

Using relation (1e), the fluid mass flow rate in the pumping direction $\psi$ (Figures 4 and 5), further denoted by $\dot{M}_{\psi}$, can be expressed by the integral representation $[3,5]$ :

$$
\dot{\mathscr{M}}_{\psi} \cong \int_{\theta}^{\theta+\Delta \theta} r_{0} \sin \beta_{0} d \theta \int_{0}^{h} \rho v_{\psi}(y) d y,
$$

where $\rho$ is the fluid density and $r_{0}$ is the "reference" radius $\left(r_{0} \in\left[r_{i}, \ldots, r_{e}\right]\right.$, and further $r_{0}$ will be denoted by $\left.r\right)$.

\section{Differential Equation for Pressure Distribution in the $\psi$ Direction}

Observing the bearing geometry (Figures 1 and 2), we admit that the angle $\Delta \theta$ is infinitely small, meaning that there exist an infinite number of spiral channels. Given the relations (1e) and (3), the physical natural condition is that the mass flow rate $\dot{M}_{\psi}$ is constant. With these conditions we obtain

$$
\dot{\mathscr{M}}_{\psi} \cong \Delta \theta\left[C \frac{\partial p}{\partial \psi}+D r^{2}\right]
$$

where

$$
\begin{gathered}
C=-\frac{\rho}{12 \eta}\left[\alpha h_{1}^{3}+(1-\alpha) h_{2}^{3}\right] \\
D=\frac{\rho}{2} \omega_{1} \sin \beta_{0} \cos \beta_{0}\left[\alpha h_{1}+(1-\alpha) h_{2}\right] .
\end{gathered}
$$

The fluid mass conservation in the $\psi$ direction can be expressed as follows:

$$
\frac{\partial}{\partial \tau}(m)+\frac{\partial}{\partial \psi}\left(\dot{M}_{\psi}\right) \Delta \psi \cong 0
$$

where $\tau$ is the time, and the fluid mass $m$, contained in the control volume Vol, is given by the relation

$$
m \cong \Delta r \Delta \theta \rho r\left[\alpha h_{1}+(1-\alpha) h_{2}\right] .
$$

Or, using relations (4) and (7), (6) becomes

$$
\begin{aligned}
& \frac{\partial}{\partial \tau}\left\{\left[\alpha h_{1}+(1-\alpha) h_{2}\right] \rho\right\} \\
& \quad+\sin ^{2} \beta_{0} \frac{1}{\psi} \frac{\partial}{\partial \psi}\left\{C \frac{\partial p}{\partial \psi}+D \frac{1}{\sin ^{2} \beta_{0}} \psi^{2}\right\} \cong 0 .
\end{aligned}
$$

For the permanent motion regime, (8) becomes

$$
\frac{1}{\psi} \frac{\partial}{\partial \psi}\left\{C \frac{\partial p}{\partial \psi}+\frac{1}{\sin ^{2} \beta_{0}} D \psi^{2}\right\} \cong 0 .
$$

Using nondimensional variables $[1,3-5,7,10,14,15]$, (8) can be written as

$$
\frac{\partial}{\partial \zeta}\left[P H^{3}\left(K_{1} \zeta \frac{\partial P}{\partial \zeta}+K_{2} \Omega \Lambda \zeta^{2} H^{-2}\right)\right]-\sigma \zeta \frac{\partial}{\partial t}\left[P H K_{3}\right] \cong 0 .
$$

For the stationary motion regime, $(10)$ becomes $[1,2]$

$$
\frac{\partial}{\partial \zeta}\left[P\left(K_{1} \zeta \frac{\partial P}{\partial \zeta}+K_{2} \Omega \Lambda \zeta^{2}\right)\right] \cong 0
$$

\section{Integration of the Differential Equation of the Pressure Distribution in the $\psi$ Direction}

In the $\psi$ direction, the fluid film $h$ varies rapidly from $h_{1}$ to $h_{2}$, at the frontier $r \cong r_{c}$ (Figures 4 and 5). The existing radial step with length in the pumping direction $\psi$ produces a pressure jump from $p_{h 1}$ to $p_{h 2}$. This pressure jump has different values as a function of (i) the flow regime through bearing (laminar, transition, or turbulent regime), (ii) the value of the rapport $h_{1} / h_{2}$, (iii) the fact that we take (or not) into consideration the inertial forces, and (iv) the fluid type $[2,3,5,16,17]$. 


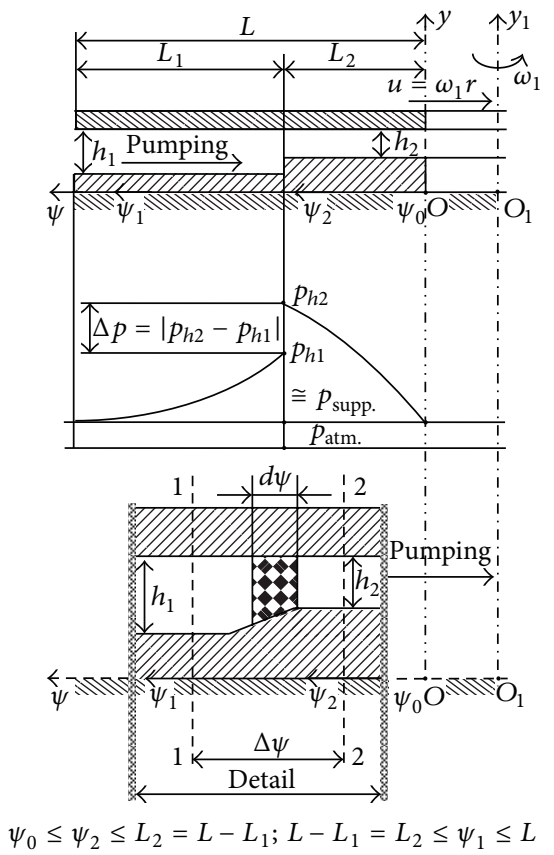

Figure 4: Radial step of the inner pumping bearing.

Integrating differential equation (9) and observing the limit conditions for pressures $[3,5]$ and the notations from Figures 4 and 5 ( $p_{\text {supp. }}$ is the supply pressure of the lubrication fluid and $p_{\text {atm }}$ is the atmospheric pressure), we obtain the mathematical relations for the pressure distributions in the SATBESPIG:

$$
\begin{aligned}
& p(\psi) \\
& \cong p_{h 2}+\frac{\psi-\left(L_{2}+\psi_{0}\right)}{\psi_{0}-\left(L_{2}+\psi_{0}\right)} \\
& \quad \times\left\{\left(p_{\text {supp. }}-p_{h 2}\right)-\frac{2 \eta \cos \beta_{0} \omega_{1}}{h_{2}^{2} \sin \beta_{0}}\left[\psi_{0}^{3}-\left(L_{2}+\psi_{0}\right)^{3}\right]\right\} \\
& \quad+\frac{2 \eta \cos \beta_{0} \omega_{1}}{h_{2}^{2} \sin \beta_{0}} \cdot\left[\psi^{3}-\left(L_{2}+\psi_{0}\right)^{3}\right] .
\end{aligned}
$$

Relation (12) presents the pressure distribution in the laminar and permanent flow regime in the smooth region of the inner pumping bearing surface, where $h=h_{2}$. Consider

$$
\begin{aligned}
& p(\psi) \\
& \cong p_{\text {supp. }}+\frac{\psi-\left(L+\psi_{0}\right)}{\left(L_{2}+\psi_{0}\right)-\left(L+\psi_{0}\right)} \\
& \quad \times\left\{\left(p_{h 1}-p_{\text {supp. }}\right)-\frac{2 \eta \cos \beta_{0} \omega_{1}\left[\alpha h_{1}+(1-\alpha) h_{2}\right]}{\sin \beta_{0}\left[\alpha h_{1}^{3}+(1-\alpha) h_{2}^{3}\right]}\right.
\end{aligned}
$$

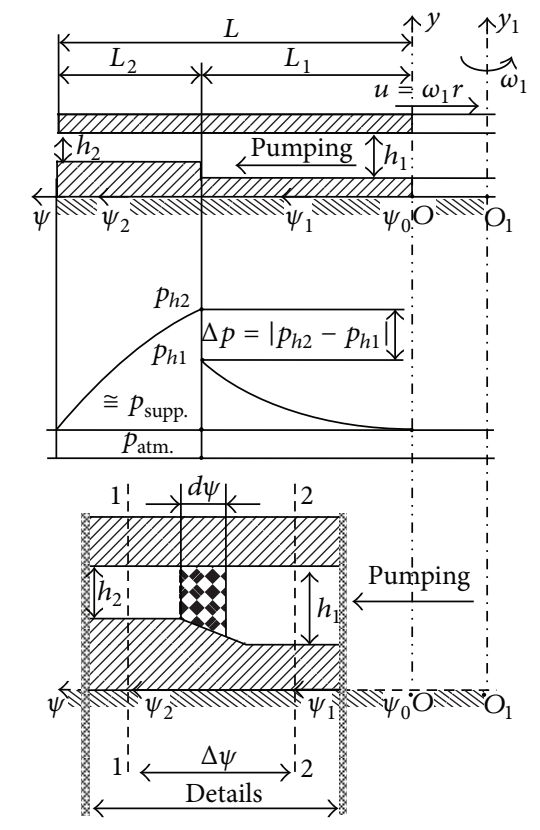

$$
\psi_{0} \leq \psi_{1} \leq L_{1}=L-L_{2} ; L-L_{2}=L_{1} \leq \psi_{2} \leq L
$$

FIGURE 5: Radial step of the external pumping bearing.

$$
\begin{aligned}
\left.\times\left[\left(L_{2}+\psi_{0}\right)^{3}-\left(L+\psi_{0}\right)^{3}\right]\right\} \\
+\frac{2 \eta \cos \beta_{0} \omega_{1}\left[\alpha h_{1}+(1-\alpha) h_{2}\right]}{\sin \beta_{0}\left[\alpha h_{1}^{3}+(1-\alpha) h_{2}^{3}\right]}\left[\psi^{3}-\left(L+\psi_{0}\right)^{3}\right] .
\end{aligned}
$$

Relation (13) presents the pressure distribution in the region with spiral channels of the inner pumping bearing surface, where $h=h_{1}$.

In a similar way, for the SATBESPIG with exterior pumping (Figure 5), we obtain

$$
\begin{aligned}
& p(\psi) \\
& \cong p_{h 2}+\frac{\psi-\left(L_{1}+\psi_{0}\right)}{\left(L+\psi_{0}\right)-\left(L_{1}+\psi_{0}\right)} \\
& \quad \times\left\{\left(p_{\text {supp. }}-p_{h 2}\right)-\frac{2 \eta \cos \beta_{0} \omega_{1}}{h_{2}^{2} \sin \beta_{0}}\left[\left(L+\psi_{0}\right)^{3}-\left(L_{1}+\psi_{0}\right)^{3}\right]\right\} \\
& +\frac{2 \eta \cos \beta_{0} \omega_{1}}{h_{2}^{2} \sin \beta_{0}}\left[\psi^{3}-\left(L_{1}+\psi_{0}\right)^{3}\right] .
\end{aligned}
$$


Relation (14) presents the pressure distribution in the laminar and permanent flow regime in the smooth region of the external pumping bearing surface, where $h=h_{2}$. Consider

$$
\begin{aligned}
& p(\psi) \\
& \cong p_{\text {supp. }}+\frac{\psi-\psi_{0}}{\left(L_{1}+\psi_{0}\right)-\psi_{0}} \\
& \quad \times\left\{\left(p_{h 1}-p_{\text {supp. }}\right)-\frac{2 \eta \cos \beta_{0} \omega_{1}\left[\alpha h_{1}+(1-\alpha) h_{2}\right]}{\sin \beta_{0}\left[\alpha h_{1}^{3}+(1-\alpha) h_{2}^{3}\right]}\right. \\
& \left.\quad \times\left[\left(L_{1}+\psi_{0}\right)^{3}-\psi_{0}^{3}\right]\right\} \\
& +\frac{2 \eta \cos \beta_{0} \omega_{1}\left[\alpha h_{1}+(1-\alpha) h_{2}\right]}{\sin \beta_{0}\left[\alpha h_{1}^{3}+(1-\alpha) h_{2}^{3}\right]}\left[\psi^{3}-\psi_{0}^{3}\right] .
\end{aligned}
$$

Relation (15) presents the pressure distribution in the laminar and permanent motion regime in the spiral grooves region of the bearing surface with exterior pumping, where $h=h_{1}$.

In the relations (12)-(15), all the constants are known (for a designed and realized SATBESPIG), the exception being the extreme pressures $p_{h 1}$ and $p_{h 2}$. If we do not take into consideration the influence of the inertial forces, then the pressures $p_{h 1}$ and $p_{h 2}$ are equal. Thus, $p_{h 1} \equiv p_{h 2}, \Delta p=$ $p_{h 2}-p_{h 1}=0$.

\section{Calculus Relations for the Extreme Pressures $p_{h 1}$ and $p_{h 2}$}

To express the pressure distribution in the $\psi$ direction and the extreme pressures $p_{h 1}$ and $p_{h 2}$, we must analyze the liquid motion in the fluid film existing between the quasiparallel surfaces of the SATBESPIG $[1-3,5,7]$. On the other hand, the inertial effects (which considerably influence the extreme pressures $p_{h 1}$ and $p_{h 2}$ ) exist on all the surfaces of the SATBESPIG in the $\psi$ direction, but the maximum effect is concentrated in the zone of the radial step, $r \cong r_{c}$ (Figures 4 and 5) $[2,3,5,16,17]$.

Some theoretical results concerning the motion of liquids in similar bearings with the consideration of the influence of inertial forces have been presented in the literature $[3,5-7$, 14]. It is possible to demonstrate $[3,5]$ that, for the case of the stationary motion regime and when only the smooth surface is in a rotation with $n_{1}=$ constant [rot $/ \mathrm{min}$ ], the equation that describes the viscous fluid motion in $\psi$ direction is

$$
\begin{aligned}
& \frac{\rho}{\psi} \frac{d}{d \psi}\left(\frac{\alpha_{0} Q_{\psi}^{2}}{h \psi^{2} \Delta \theta^{2}}-\frac{\gamma Q_{\psi} \omega_{1}}{\Delta \theta} \frac{\cos \beta_{0}}{\sin \beta_{0}}+\beta \omega_{1}^{2} \psi^{2} \frac{\cos ^{2} \beta_{0}}{\sin ^{2} \beta_{0}} h\right) \\
& +\frac{2 \rho \delta Q_{\psi} \omega_{1} \sin \beta_{0}}{\psi^{2} \Delta \theta \cos \beta_{0}}-3 \rho \beta \omega_{1}^{2} h+\frac{\rho \alpha_{0} Q_{\psi}^{2}}{h \psi^{4} \Delta \theta^{2}}
\end{aligned}
$$

$$
\begin{aligned}
& -\frac{\rho \gamma Q_{\psi} \omega_{1} \cos \beta_{0}}{\psi^{2} \Delta \theta \sin \beta_{0}}+\rho \beta \omega_{1}^{2} \frac{\cos ^{2} \beta_{0}}{\sin ^{2} \beta_{0}} h+\frac{1}{\psi} \frac{d p}{d \psi} h \\
& +\frac{12 \eta}{h}\left(\frac{Q_{\psi}}{h \psi \Delta \theta}-\frac{\omega_{1} \psi \cos \beta_{0}}{2 \sin \beta_{0}}\right) \cong 0
\end{aligned}
$$

where

$$
\begin{aligned}
Q_{\psi}=U_{\text {av. }} h \cong & -\frac{h^{3}}{12 \eta r \sin \beta_{0}} \frac{\partial p}{\partial \psi} \\
& +\frac{h}{2} \omega_{1} r \cos \beta_{0}=\text { const. (volumic flow rate), }
\end{aligned}
$$

$U_{\text {av }}$

$=\frac{1}{h} \int_{0}^{h} v_{\psi}(y) \cdot d y$

$=$ average speed in the fluid film by the curb direction $\psi$,

$$
\alpha_{0}=\frac{6}{5}, \quad \beta=\frac{2}{15}, \quad \gamma=\frac{1}{5}, \quad \delta=\frac{1}{10} \text {. }
$$

The constructive angle of spiral groove is $\beta_{0}=17^{\circ}[3,5,14]$.

Calculating the derivative of the relation (16) versus the variable $\psi$ and taking into consideration the constants from above, the following expression can be found:

$$
\begin{aligned}
\frac{d p}{d \psi} \cong & \left(\frac{\rho \alpha_{0} Q_{\psi}^{2}}{h^{3} \psi^{2} \Delta \theta^{2}}-\frac{\rho \beta \omega_{1}^{2} \psi^{2}}{h} \frac{\cos ^{2} \beta_{0}}{\sin ^{2} \beta_{0}}\right) \frac{d h}{d \psi}+\frac{\rho \alpha_{0} Q_{\psi}^{2}}{h^{2} \psi^{3} \Delta \theta^{2}} \\
& +3 \rho \beta \omega_{1}^{2} \psi\left(1-\frac{\cos ^{2} \beta_{0}}{\sin ^{2} \beta_{0}}\right)-\frac{2 \rho \delta Q_{\psi} \omega_{1} \sin \beta_{0}}{h \psi \Delta \theta \cos \beta_{0}} \\
& +\frac{\rho \gamma Q_{\psi} \omega_{1} \cos \beta_{0}}{h \psi \Delta \theta \sin \beta_{0}}-\frac{12 \eta Q_{\psi}}{h^{3} \Delta \theta}+\frac{6 \eta \omega_{1} \psi^{2} \cos \beta_{0}}{h^{2} \sin \beta_{0}} .
\end{aligned}
$$

We obtain a similar but more precise relation to (18) by introducing a supplementary coercive term $[1,2,4,9]$. In this case, relation (18) becomes

$$
\begin{aligned}
\frac{d p}{d \psi} \cong & {\left[\frac{\rho Q_{\psi}^{2}}{h^{3} \psi^{2} \Delta \theta^{2}}\left(\alpha_{0}+\varepsilon \psi\right)\right] \frac{d h}{d \psi}-\frac{\rho \beta \omega_{1}^{2} \psi^{2}}{h} \frac{\cos ^{2} \beta_{0}}{\sin ^{2} \beta_{0}} \frac{d h}{d \psi} } \\
& +\frac{\rho \alpha_{0} Q_{\psi}^{2}}{h^{2} \psi^{3} \Delta \theta^{2}}+3 \rho \beta \omega_{1}^{2} \psi\left(1-\frac{\cos ^{2} \beta_{0}}{\sin ^{2} \beta_{0}}\right) \\
& -\frac{2 \rho \delta Q_{\psi} \omega_{1} \sin \beta_{0}}{h \psi \Delta \theta \cos \beta_{0}}+\frac{\rho \gamma Q_{\psi} \omega_{1} \cos \beta_{0}}{h \psi \Delta \theta \sin \beta_{0}}-\frac{12 \eta Q_{\psi}}{h^{3} \Delta \theta} \\
& +\frac{6 \eta \omega_{1} \psi^{2} \cos \beta_{0}}{h^{2} \sin \beta_{0}}
\end{aligned}
$$

where $\varepsilon=2 / 15[1,2,4]$. 
Integrating the differential equation (19), for both regions of the SATBESPIG, where $h=h_{1}=$ const. and $h=h_{2}=$ const. (Figures 4 and 5), we obtain the calculus relations for the pressures $p_{h 1}$ and $p_{h 2}$ :

$$
\begin{aligned}
& p_{h 1} \cong p_{\text {supp. }}+\frac{\rho \alpha_{0} Q_{\psi}^{2}}{2 h_{1}^{2} \Delta \theta^{2}}\left[\frac{1}{\left(L+\psi_{0}\right)^{2}}-\frac{1}{\left(L_{2}+\psi_{0}\right)^{2}}\right] \\
& +\frac{3}{2} \rho \beta \omega_{1}^{2}\left(1-\frac{\cos ^{2} \beta_{0}}{\sin ^{2} \beta_{0}}\right)\left[\left(L_{2}+\psi_{0}\right)^{2}-\left(L+\psi_{0}\right)^{2}\right] \\
& -\frac{2 \rho \delta Q_{\psi} \omega_{1} \sin \beta_{0}}{h_{1} \Delta \theta \cos \beta_{0}} \ln \frac{L_{2}+\psi_{0}}{L+\psi_{0}} \\
& +\frac{\rho \gamma Q_{\psi} \omega_{1} \cos \beta_{0}}{h_{1} \Delta \theta \sin \beta_{0}} \ln \frac{L_{2}+\psi_{0}}{L+\psi_{0}} \\
& +\frac{12 \eta Q_{\psi}}{h_{1}^{3} \Delta \theta}\left[\left(L+\psi_{0}\right)-\left(L_{2}+\psi_{0}\right)\right] \\
& -\frac{2 \eta \omega_{1} \cos \beta_{0}}{h_{1}^{2} \sin \beta_{0}} \cdot\left[\left(L+\psi_{0}\right)^{3}-\left(L_{2}+\psi_{0}\right)^{3}\right], \\
& p_{h 2} \cong p_{\text {supp. }}-\frac{\rho \alpha_{0} Q_{\psi}^{2}}{2 h_{2}^{2} \Delta \theta^{2}}\left[\frac{1}{\left(L_{2}+\psi_{0}\right)^{2}}-\frac{1}{\psi_{0}^{2}}\right] \\
& +\frac{3}{2} \rho \beta \omega_{1}^{2}\left(1-\frac{\cos ^{2} \beta_{0}}{\sin ^{2} \beta_{0}}\right)\left[\left(L_{2}+\psi_{0}\right)^{2}-\psi_{0}^{2}\right] \\
& +\frac{2 \rho \delta Q_{\psi} \omega_{1} \sin \beta_{0}}{h_{2} \Delta \theta \cos \beta_{0}} \ln \frac{\psi_{0}}{L_{2}+\psi_{0}} \\
& -\frac{\rho \gamma Q_{\psi} \omega_{1} \cos \beta_{0}}{h_{2} \Delta \theta \sin \beta_{0}} \ln \frac{\psi_{0}}{L_{2}+\psi_{0}} \\
& +\frac{12 \eta Q_{\psi}}{h_{2}^{3} \Delta \theta}\left[\psi_{0}-\left(L_{2}+\psi_{0}\right)\right] \\
& +\frac{2 \eta \omega_{1} \cos \beta_{0}}{h_{2}^{2} \sin \beta_{0}}\left[\left(L_{2}+\psi_{0}\right)^{3}-\psi_{0}^{3}\right] \text {. }
\end{aligned}
$$

These relations are valid for the SATBESPIG with inner pumping.

In a similar way, for the SATBESPIG with exterior pumping, we obtain

$$
\begin{aligned}
p_{h 1} \cong & p_{\text {supp. }}+\frac{\rho \alpha_{0} Q_{\psi}^{2}}{2 h_{1}^{2} \Delta \theta^{2}}\left[\frac{1}{\psi_{0}^{2}}-\frac{1}{\left(L_{1}+\psi_{0}\right)^{2}}\right] \\
& +\frac{3}{2} \rho \beta \omega_{1}^{2}\left(1-\frac{\cos ^{2} \beta_{0}}{\sin ^{2} \beta_{0}}\right)\left[\left(L_{1}+\psi_{0}\right)^{2}-\psi_{0}^{2}\right] \\
& +\frac{2 \rho \delta Q_{\psi} \omega_{1} \sin \beta_{0}}{h_{1} \Delta \theta \cos \beta_{0}} \ln \frac{\psi_{0}}{L_{1}+\psi_{0}}
\end{aligned}
$$

$$
\begin{aligned}
&-\frac{\rho \gamma Q_{\psi} \omega_{1} \cos \beta_{0}}{h_{1} \Delta \theta \sin \beta_{0}} \ln \frac{\psi_{0}}{L_{1}+\psi_{0}} \\
&+\frac{12 \eta Q_{\psi}}{h_{1}^{3} \Delta \theta}\left[\psi_{0}-\left(L_{1}+\psi_{0}\right)\right] \\
&-\frac{2 \eta \omega_{1} \cos \beta_{0}}{h_{1}^{2} \sin \beta_{0}}\left[\psi_{0}^{3}-\left(L_{1}+\psi_{0}\right)^{3}\right] \\
& p_{h 2}+p_{\text {supp. }}+\frac{\rho \alpha_{0} Q_{\psi}^{2}}{2 h_{2}^{2} \Delta \theta^{2}}\left[\frac{1}{\left(L+\psi_{0}\right)^{2}}-\frac{1}{\left(L_{1}+\psi_{0}\right)^{2}}\right] \\
&+\frac{3}{2} \rho \beta \omega_{1}^{2}\left(1-\frac{\cos ^{2} \beta_{0}}{\sin ^{2} \beta_{0}}\right)\left[\left(L_{1}+\psi_{0}\right)^{2}-\left(L+\psi_{0}\right)^{2}\right] \\
&-\frac{2 \rho \delta Q_{\psi} \omega_{1} \sin \beta_{0}}{h_{2} \Delta \theta \cos \beta_{0}} \ln \frac{L_{1}+\psi_{0}}{L+\psi_{0}} \\
&+\frac{\rho \gamma Q_{\psi} \omega_{1} \cos \beta_{0}}{h_{2} \Delta \theta \sin \beta_{0}} \ln \frac{L_{1}+\psi_{0}}{L+\psi_{0}} \\
&+ \frac{12 \eta Q_{\psi}}{h_{2}^{3} \Delta \theta}\left[\left(L+\psi_{0}\right)-\left(L_{1}+\psi_{0}\right)\right] \\
&+\frac{2 \eta \sin \beta_{0}}{h_{2}^{2}}\left[\left(L_{1}+\psi_{0}\right)^{3}-\left(L+\psi_{0}\right)^{3}\right] \\
&
\end{aligned}
$$

In relations (20)-(22), all the variables are known except the volumetric flow rate $Q_{\psi}$.

\section{Calculus Relation for the Fluid Volumetric Flow Rate $Q_{\psi}$}

The calculus relation for the fluid volumetric flow rate $Q_{\psi}$ will be established using differential equation (19) again. To find the calculus relation, at the position of the radial step of the bearing we suppose that $d h / d \psi \neq 0$. So, in this zone of the bearing the inertial effects are dominant in comparison to the viscous effects. In other words, in the radial step zone of the bearing, the liquid moves approximately like an ideal but not viscous fluid $[3,5]$.

Integrating (19) in the vicinity of the radial step of the bearing grooved surface (Figures 4 and 5), we obtain a relation between $p_{h 1}, p_{h 2}$, and $Q_{\psi}$ :

$$
\begin{aligned}
\Delta p=p_{h 1}-p_{h 2} \cong & \frac{\rho Q_{\psi}^{2}}{\psi_{c}^{2} \Delta \theta^{2}}\left(\alpha_{0}+\varepsilon \psi_{c}\right)\left(\frac{1}{2 h_{2}^{2}}-\frac{1}{2 h_{1}^{2}}\right) \\
& -\rho \beta \omega_{1}^{2} \psi_{c}^{2} \frac{\cos ^{2} \beta_{0}}{\sin ^{2} \beta_{0}} \ln \frac{h_{1}}{h_{2}},
\end{aligned}
$$

where $\psi_{c}$ is the length measured by the $\psi$ coordinate corresponding to the grooves radius of the profiled surface, $r_{c}$ (Figures 4 and 5).

Relation (23) has some limits, especially at high values of $h_{1} / h_{2}$, when $h_{2} \rightarrow 0$, or, in other words, at the heavy regimes 
for the bearing functionality. If we know the other variables, including the extreme pressures $p_{h 1}$ and $p_{h 2}$, relation (23) offers the flow rate $Q_{\psi}$.

Therefore, using (20) and then (21), (22), and (23), we obtain a typical second degree algebraic equation, (24), from which we obtain the fluid flow rate $Q_{\psi}$ :

$$
\begin{gathered}
\frac{\rho Q_{\psi}^{2}}{\Delta \theta^{2}}\left\{\alpha _ { 0 } \left[\frac{1}{2 h_{1}^{2}}\left(\frac{1}{\left(L+\psi_{0}\right)^{2}}-\frac{1}{\left(L_{2}+\psi_{0}\right)^{2}}\right)\right.\right. \\
\left.\quad+\frac{1}{2 h_{2}^{2}}\left(\frac{1}{\left(L_{2}+\psi_{0}\right)^{2}}-\frac{1}{\psi_{0}^{2}}\right)\right] \\
\left.+\frac{1}{\psi_{c}^{2}}\left(\alpha_{0}+\varepsilon \psi_{c}\right)\left(\frac{1}{2 h_{2}^{2}}-\frac{1}{2 h_{1}^{2}}\right)\right\} \\
\quad\left[\frac{\rho \gamma \omega_{1} \cos \beta_{0}}{\sin \beta_{0}\left(\frac{1}{h_{1}} \ln \frac{L_{2}+\psi_{0}}{L+\psi_{0}}+\frac{1}{h_{2}} \ln \frac{\psi_{0}}{L_{2}+\psi_{0}}\right)}\right. \\
\quad-\frac{2 \rho \delta \omega_{1} \sin \beta_{0}}{\cos \beta_{0}}\left(\frac{1}{h_{1}} \ln \frac{L_{2}+\psi_{0}}{L+\psi_{0}}+\frac{1}{h_{2}} \ln \frac{\psi_{0}}{L_{2}+\psi_{0}}\right) \\
\left.+12 \eta\left(\frac{L-L_{2}}{h_{1}^{3}}+\frac{L_{2}}{h_{2}^{3}}\right)\right]-T 1+T 2 \cong 0,
\end{gathered}
$$

where

$$
\begin{gathered}
T 1=\frac{3}{2} \rho \beta \omega_{1}^{2}\left(1-\frac{\cos ^{2} \beta_{0}}{\sin ^{2} \beta_{0}}\right)\left[\left(L+\psi_{0}\right)^{2}-\psi_{0}^{2}\right], \\
T 2=\rho \beta \omega_{1}^{2} \psi_{c}^{2} \frac{\cos ^{2} \beta_{0}}{\sin ^{2} \beta_{0}} \ln \frac{h_{1}}{h_{2}}-\frac{2 \eta \omega_{1} \cos \beta_{0}}{\sin \beta_{0}} \\
\times\left\{\frac{1}{h_{1}^{2}}\left[\left(L+\psi_{0}\right)^{3}-\left(L_{2}+\psi_{0}\right)^{3}\right]\right. \\
\left.+\frac{1}{h_{2}^{2}}\left[\left(L_{2}+\psi_{0}\right)^{3}-\psi_{0}^{3}\right]\right\} .
\end{gathered}
$$

Relation (24) is valid for the SATBESPIG with inner pumping.

For the SATBESPIG with exterior pumping, we obtain a similar relation:

$$
\begin{gathered}
\frac{\rho Q_{\psi}^{2}}{\Delta \theta^{2}}\left\{\alpha _ { 0 } \left[\frac{1}{2 h_{2}^{2}}\left(\frac{1}{\left(L+\psi_{0}\right)^{2}}-\frac{1}{\left(L_{1}+\psi_{0}\right)^{2}}\right)\right.\right. \\
\left.\quad-\frac{1}{2 h_{1}^{2}}\left(\frac{1}{\psi_{0}^{2}}-\frac{1}{\left(L_{1}+\psi_{0}\right)^{2}}\right)\right] \\
\left.+\frac{1}{2 \psi_{c}^{2}}\left(\alpha_{0}+\varepsilon \psi_{c}\right)\left(\frac{1}{h_{2}^{2}}-\frac{1}{h_{1}^{2}}\right)\right\}
\end{gathered}
$$

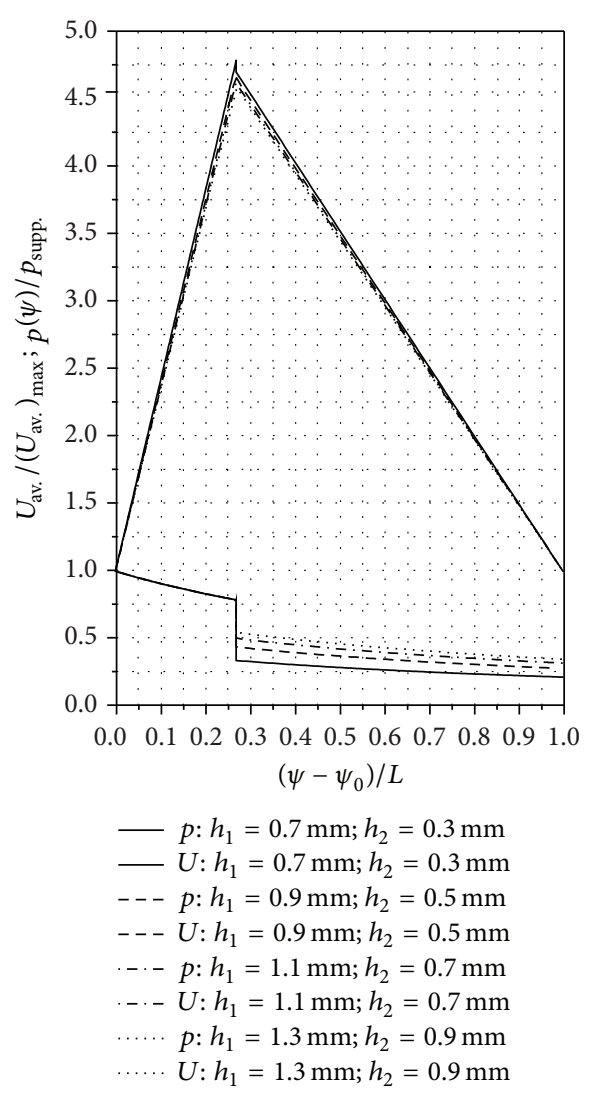

FIGURE 6: Pressure and speed distributions in the SATBESPIG with inner pumping. (See details in Figures 7 and 8.)

$$
\begin{aligned}
+\frac{Q_{\psi}}{\Delta \theta} \cdot[ & \frac{\rho \gamma \omega_{1} \cos \beta_{0}}{\sin \beta_{0}}\left(\frac{1}{h_{1}} \ln \frac{\psi_{0}}{L_{1}+\psi_{0}}+\frac{1}{h_{2}} \ln \frac{L_{1}+\psi_{0}}{L+\psi_{0}}\right) \\
& -\frac{2 \rho \delta \omega_{1} \sin \beta_{0}}{\cos \beta_{0}}\left(\frac{1}{h_{1}} \ln \frac{\psi_{0}}{L_{1}+\psi_{0}}+\frac{1}{h_{2}} \ln \frac{L_{1}+\psi_{0}}{L+\psi_{0}}\right) \\
+ & \left.12 \eta\left(\frac{L-L_{1}}{h_{2}^{3}}+\frac{L_{1}}{h_{1}^{3}}\right)\right]-T 1-T 3 \cong 0
\end{aligned}
$$

where

$$
\begin{aligned}
T 3=\rho \beta \omega_{1}^{2} \psi_{c}^{2} \frac{\cos ^{2} \beta_{0}}{\sin ^{2} \beta_{0}} \ln \frac{h_{1}}{h_{2}} \\
-\frac{2 \eta \omega_{1} \cos \beta_{0}}{\sin \beta_{0}}\left\{\frac{1}{h_{1}^{2}}\left[\psi_{0}^{3}-\left(L_{1}+\psi_{0}\right)^{3}\right]\right. \\
\left.+\frac{1}{h_{2}^{2}}\left[\left(L_{1}+\psi_{0}\right)^{3}-\left(L+\psi_{0}\right)^{3}\right]\right\} .
\end{aligned}
$$

Both (24) and (28) are classical algebraic equations of second degree in $Q_{\psi}$. If we denote by $Q_{\psi}^{I}$ and $Q_{\psi}^{I I}$ the two solutions of the every algebraic equation (24) or (28), using $Q_{\psi}^{I}$ and $Q_{\psi}^{I I}$, and if we take into consideration that the 


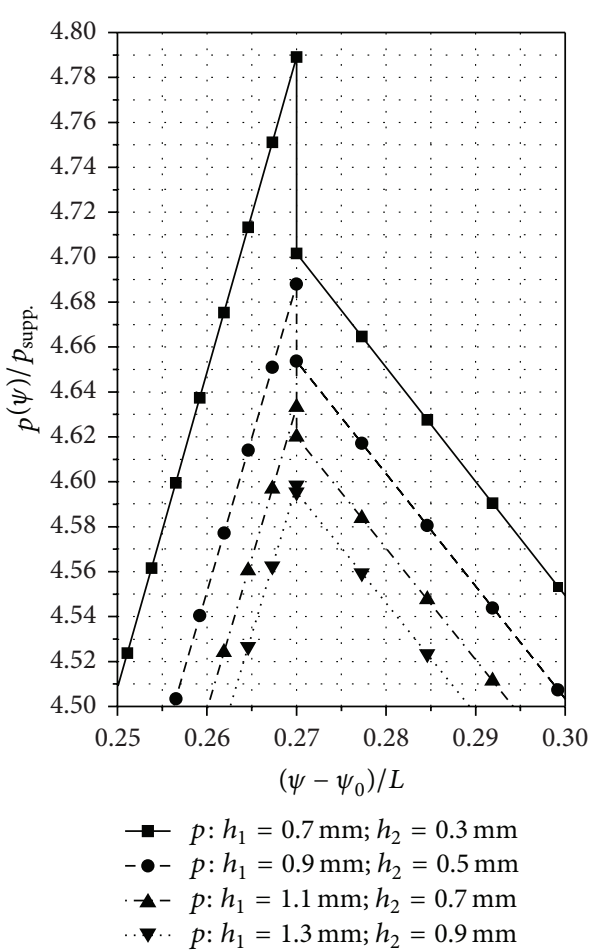

FIGURE 7: Detail of Figure 6 concerning the pressure jump between $h_{1}$ and $h_{2}$. (The marked points are those where we made the calculation.)

SATBESPIG realizes inner pumping and exterior pumping, it is not possible to have a negative fluid flow rate from a physical point of view. Thus, the algebraic solution, which has physical meaning, is the positive solution $[3,5]$.

The numerical evaluation of $Q_{\psi}^{I}$ and $Q_{\psi}^{I I}$ algebraic solutions, for $Q_{\psi}^{I}$ and $Q_{\psi}^{I I}$ using medium (normal) values for the physical and geometrical dimensions $[1,2]$ of $(24)$ and $(28)$, leads to $Q_{\psi}^{I}<0$ and $Q_{\psi}^{I I}>0$. Thus, the mathematical relation for the calculus of the fluid volumetric flow rate $Q_{\psi}$ is

$$
Q_{\psi} \equiv Q_{\psi}^{I I}
$$

where, in conformity to the devoted notations from the classical algebra, the solution $Q_{\psi}^{I I}$ is

$$
Q_{\psi}^{I I}=\frac{-\ddot{b}-\sqrt{\ddot{\ddot{b}^{2}-4 \ddot{a} \ddot{c}}}}{2 \ddot{a}}
$$

where $\ddot{a}, \ddot{b}$, and $\ddot{c}$ are the coefficients of the algebraic equations (24) or (28).

\section{Numerical and Experimental Results}

The established mathematical relations allow the numerical calculation of the pressure and speed distributions for several SATBESPIG with inner or external pumping in permanent and laminar regimes. For these numerical calculations we

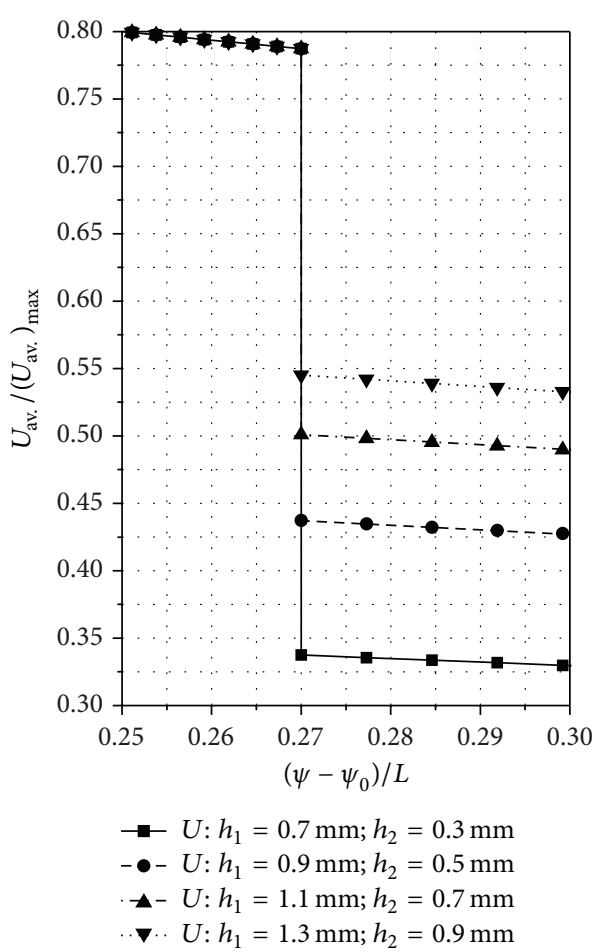

FIgURE 8: Detail of Figure 6 concerning the speed jump between $h_{1}$ and $h_{2}$. (The marked points are those where we made the calculation.)

used two different computer programs: one for the SATBESPIG with inner pumping and the other for the SATBESPIG with external pumping.

In Figures 6 to 8, we present the calculated pressure and speed distributions for a SATBESPIG with inner pumping and $r_{e}=90 \mathrm{~mm}, r_{c}=57.15 \mathrm{~mm}, r_{i}=45 \mathrm{~mm}, n_{p}=10, \alpha=0.615$, $\beta_{0}=17^{\circ}, \rho=905 \mathrm{~kg} / \mathrm{m}^{3}, \eta=10^{-4} \mathrm{~Pa} \cdot \mathrm{s}, p_{\text {supp. }}=101325 \mathrm{~N} / \mathrm{m}^{2}$, $n=900 \mathrm{rot} / \mathrm{min}, \alpha_{0}=6 / 5, \varepsilon=2 / 15, \gamma=1 / 5, \delta=1 / 10, \beta=2 / 15$, and the parameters $h_{1}$ and $h_{2}$ as written in the figures.

Figure 9 presents the calculated pressure and speed distributions for a SATBESPIG with external pumping with the same characteristics as the SATBESPIG with inner pumping above, with the exception that $r_{c}=77.85 \mathrm{~mm}$.

In Figure 10, we compared the theoretical results and the experimental measurements for the SATBESPIG with inner pumping and $h_{1}, h_{2}$, and $n$ written in the figure.

In all our studies, the main constructive geometrical and functional parameters for the calculation of these bearings were $h_{1}, h_{2}, n, r_{i}$, and $r_{e}[3,5]$.

\section{Discussion and Conclusions}

The analysis of the theoretical results allows some conclusions to be drawn concerning the laminar motion of incompressible fluids in some models of a SATBESPIG. We analyzed two variants of the SATBESPIG with inner pumping, which are called the First Variant and the Second Variant below. The difference between these bearings is the $r_{c}$ dimension. 


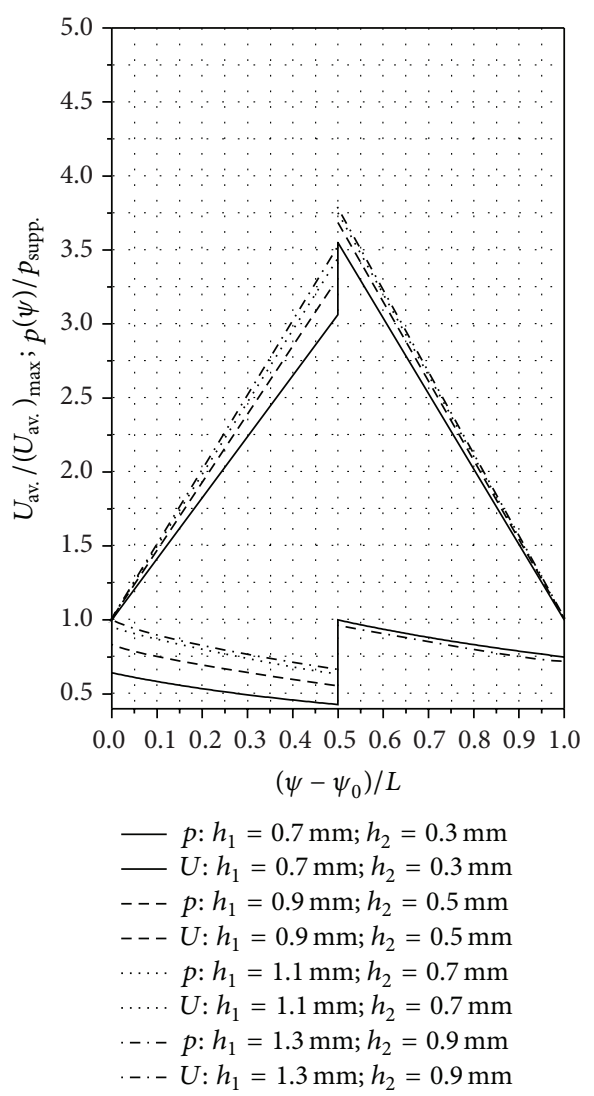

FIGURE 9: Pressure and speed distributions in the SATBESPIG with external pumping.

The First Variant is the SATBESPIG from Figure $6\left(r_{c}=\right.$ $57.15 \mathrm{~mm}$ ), and the Second Variant has $r_{c}=70.29 \mathrm{~mm}$.

(a) For all the rotation per minute (r.p.m.) ranges $n$ and for all the analyzed rapports $h_{1} / h_{2}$, the First Variant SATBESPIG with inner pumping had superior hydrodynamics performance compared to the Second Variant SATBESPIG. Thus, high pressures in bearings can be realized with an appropriate dimension of the bearing surface, especially the dimension of the grooved surface, meaning the parameters $r_{i}, r_{c}$, and $r_{e}$.

(b) Generally speaking, increasing other hydraulics performance of the SATBESPIG for the same dimensions $r_{i}, r_{c}$, and $r_{e}$ can be realized by increasing the number of the rotation per minute $n$. The modification of the rapport $h_{1} / h_{2}$ has a small influence.

(c) The nondimensional speeds increase (and decrease) from the bearing input to the bearing output, with a jump at the radial step $r \cong r_{c}$. The nondimensional pressures change, but not linearly with the channel length, from the input, where $p_{\text {imput }} \cong p_{\text {supp. }}$, up to the pressure $p_{h 1}$ and from the pressure $p_{h 2}$ to the output pressure, where $p_{\text {output }} \cong p_{\text {supp. }}$.

(d) The pressure jump $\Delta p=p_{h 1}-p_{h 2}$ theoretically tends to zero when the rapport $h_{1} / h_{2} \rightarrow 1$ and thus when

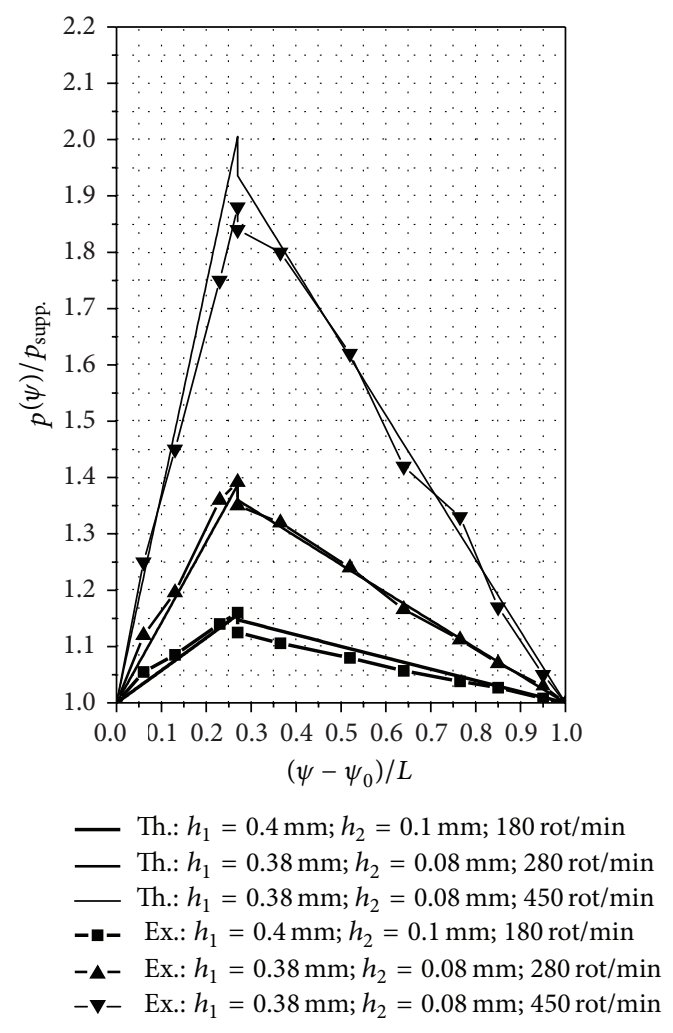

FIGURE 10: Pressure distributions in the SATBESPIG with inner pumping (comparison between the theoretical and experimental curves). The marked points are those where we performed the experimental measurements.

the radial step vanishes or when $p_{h 1} \cong p_{h 2}$. The case $p_{h 1} \cong p_{h 2}$ appears only when we do not take into consideration the inertial effects.

(e) The pressure jump $\Delta p$ depends not only on the rapport $h_{1} / h_{2}$ but also on the value of the $n$ and on the bearing geometry $\left(r_{i}, r_{c}\right.$, and $\left.r_{e}\right)$.

(f) For the same constructive geometrical and functional parameters $\left(r_{i}, r_{e}, h_{1} / h_{2}, n_{,} \ldots\right)$, the SATBESPIG with exterior pumping gives lower pressures than the bearing with inner pumping.

(g) The comparative analysis between the theoretical and experimental results shows good correlation, especially at low $n$ and at middle values for the rapport $h_{1} / h_{2}\left(h_{1} / h_{2} \cong 4\right)$. Some of the mathematical relations established above can be used to approach the theoretical functionality of the SATBESPIG having magnetic controllable fluids (magnetic fluids or magnetorheological fluids) as incompressible fluids, in the presence of a controllable magnetic field [1820].

\section{Conflict of Interests}

The authors declare that there is no conflict of interests regarding the publication of this paper. 


\section{Acknowledgment}

This work was partly supported by the Project PN II (National Education Ministry-Romania) no. 157/2012 "MagNanoMicroSeal" (2012-2015).

\section{References}

[1] V. N. Constantinescu, Al. Nica, M. D. Pascovici, G. Ceptureanu, and S. Nedelcu, Sliding Bearings, Tehnica Publishing House, Bucharest, Romania, 1980.

[2] V. N. Constantinescu, Dynamics of Viscous Fluids in Laminar Regime, Romanian Academy Publishing House, Bucharest, Romania, 1987.

[3] C. Velescu, The study of the viscous fluids motion in selfacting thrust bearings [Ph.D. thesis], "Politehnica" University of Timisoara, 1998.

[4] A. V. Yemelyanov and I. A. Yemelyanov, "Physical models, theory and fundamental improvement to self-acting spiralgrooved gas bearings and visco-seals," Proceedings of the Institution of Mechanical Engineers J, vol. 213, no. 4, pp. 263-271, 1999.

[5] C. Velescu, Hydrodynamics of the Thrust Bearings With Spiral Grooves, Mirton Publishing House, Timisoara, Romania, 2000.

[6] C. Velescu, "Theoretically remarks on the thermal and viscoinertial effects in the self acting sliding conically radiallythrust bearings," in Proceedings of the International Symposium on Fluid Machinery and Fluid Engineering (ISFMFE '96), Beijing, China, September 1996.

[7] N. Kawabata, I. Ashino, M. Sekizawa, and S. Yamazaki, "Spiral grooved bearing utilizing the pumping effect of a herringbone journal bearing. (Method of numerical calculation and influences of bearing parameters)," JSME International Journal 3, vol. 34, no. 3, pp. 411-418, 1991.

[8] S. M. Yao, "Aerostatic and aerodynamic performance of an outpump spirally grooved thrust bearing: analysis and comparisons to static load experiments," Tribology Transactions, vol. 52, no. 3, pp. 376-388, 2009.

[9] S. M. Yao, "Aerostatic and aerodynamic performance of an inpump spirally grooved thrust bearing: Analysis and comparisons to static load experiments," Transactions, vol. 51, no. 5, pp. 679-689, 2008.

[10] L. Wu, P. Liu, Z. Liu, Z. Liu, Q. Yang, and Y. Wei, “Optimization design of hydrodynamic bearing with multiple spiral grooves," in Proceedings of the 7th International Conference on Progress Machining Technology (ICPMT '04), pp. 502-507, Suzhou, China, December 2004.

[11] B. C. Majumdar, R. Pai, and D. J. Hargreaves, "Analysis of water-lubricated journal bearings with multiple axial grooves," Proceedings of the Institution of Mechanical Engineers J, vol. 218, no. 2, pp. 135-146, 2004.

[12] J. F. Zhou, B. Q. Gu, and C. Ye, "An improved design of spiral groove mechanical seal," Chinese Journal of Chemical Engineering, vol. 15, no. 4, pp. 499-506, 2007.

[13] G. I. Broman, "Implications of cavitation in individual grooves of spiral groove bearings," Proceedings of the Institution of Mechanical Engineers J, vol. 215, no. 5, pp. 417-424, 2001.

[14] S. B. Malanoski and C. H. T. Pan, "The static and dynamic characteristics of the spiral-grooved thrust bearing," Journal of Basic Engineering D-Transactions of the ASME D, vol. 87, no. 3 , pp. 547-558, 1965.
[15] W. C. Wachmann, M. S. Malanoski, and V. J. Vohr, "Thermal distortion of spiral-grooved gas-lubricated thrust bearings," Journal of Lubrication Technology-ASME Transactions, vol. 93, no. 1, pp. 102-112, 1971.

[16] C. M. Rodkiewicz and M. I. Anwar, "Inertia and convective effects in hydrodynamic lubrication of a slider bearing," Journal of Lubrication Technology F-ASME Transactions, vol. 93, 1971.

[17] C. M. Rodkiewicz, J. C. Hinds, and C. Dayson, "Inertia, convection, and dissipation effects in the thermally boosted oil lubricated sliding thrust bearing," Journal of Lubrication Technology F-ASME Transactions, vol. 97, no. 1, pp. 121-129, 1975.

[18] I. De Sabata, N. C. Popa, I. Potencz, and L. Vékás, "Inductive transducers with magnetic fluids," Sensors and Actuators A, vol. 32, no. 1-3, pp. 678-681, 1992.

[19] N. C. Popa, I. De Sabata, I. Anton, I. Potencz, and L. Vékás, "Magnetic fluids in aerodynamic measuring devices," Journal of Magnetism and Magnetic Materials, vol. 201, no. 1-3, pp. 385390, 1999.

[20] M. Liţǎ, N. C. Popa, C. Velescu, and L. N. Vékás, "Investigations of a magnetorheological fluid damper," IEEE Transactions on Magnetics, vol. 40, no. 2, pp. 469-472, 2004. 

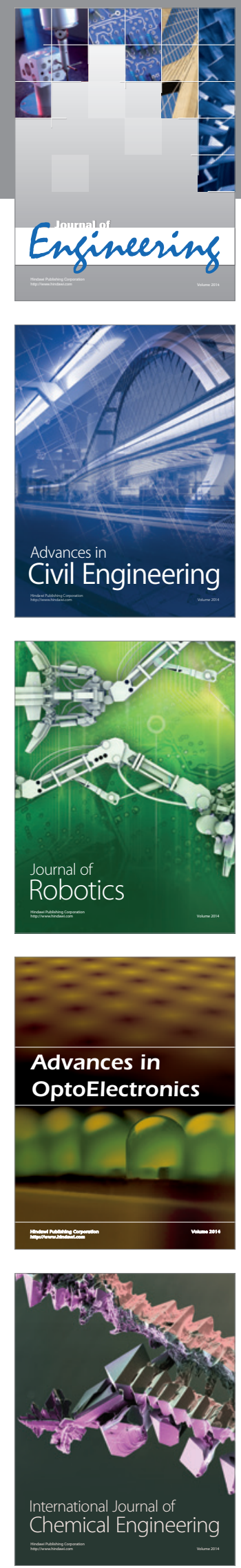

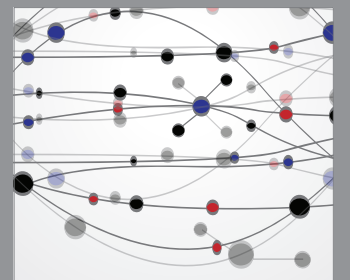

The Scientific World Journal
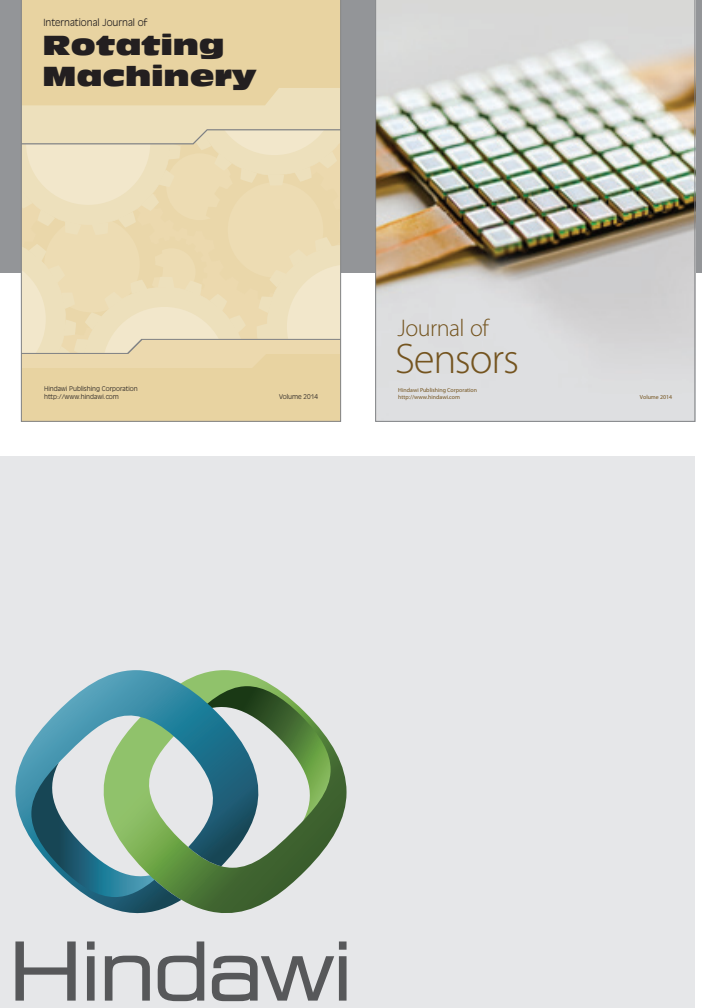

Submit your manuscripts at http://www.hindawi.com
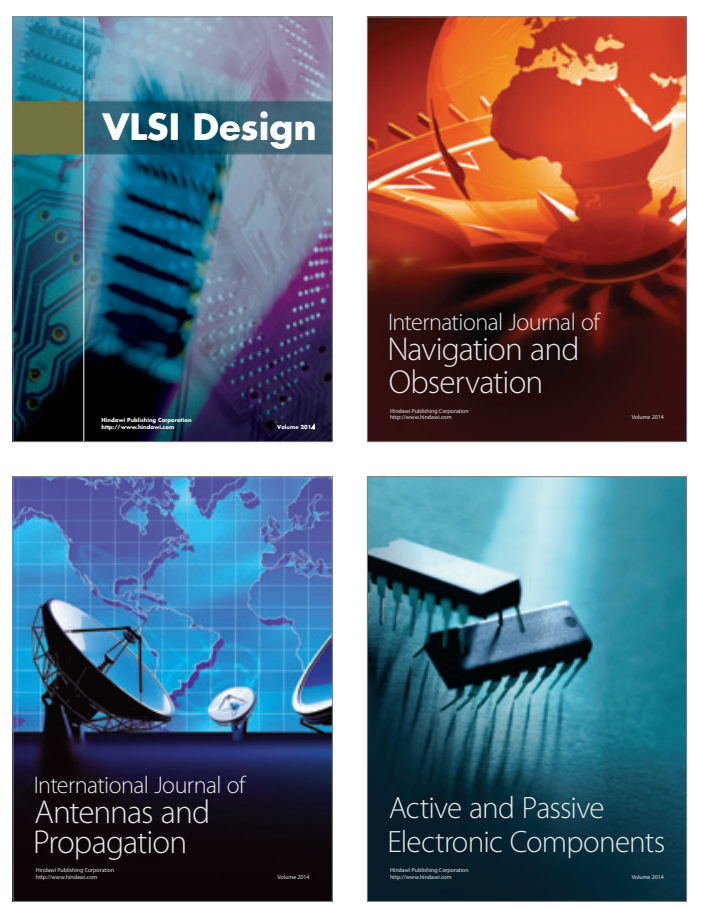
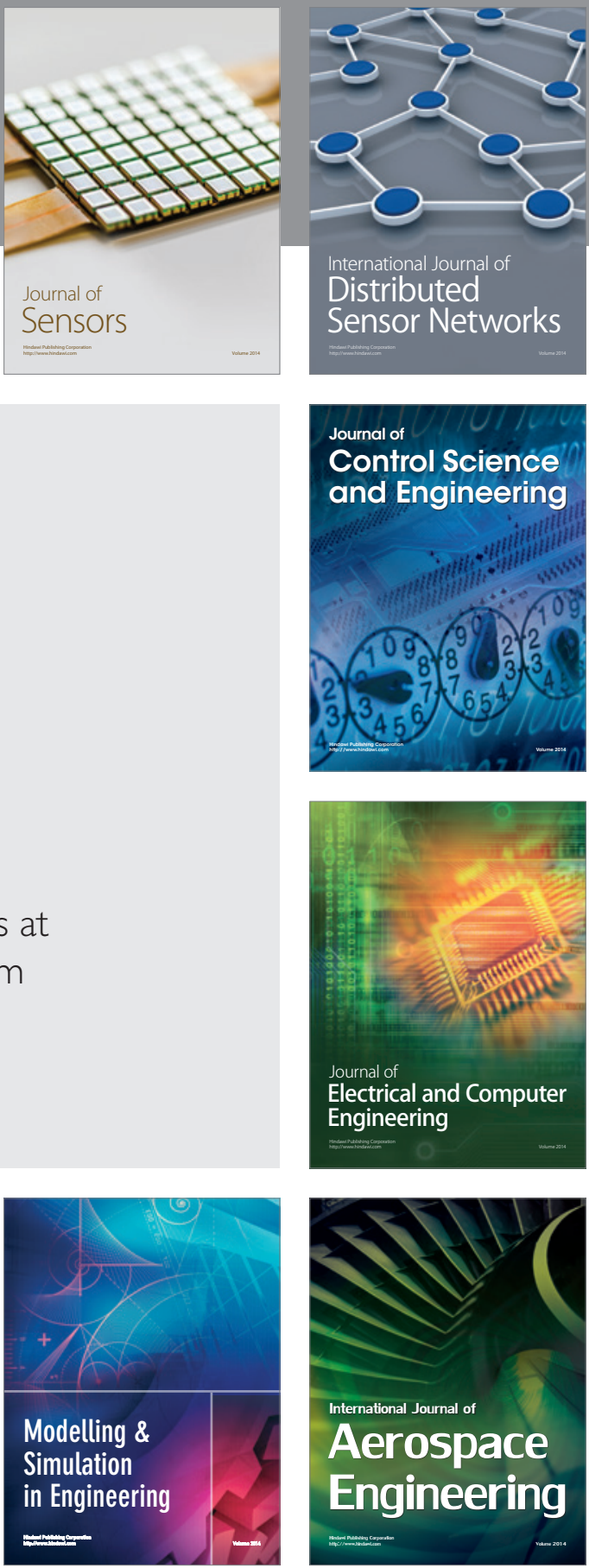

Journal of

Control Science

and Engineering
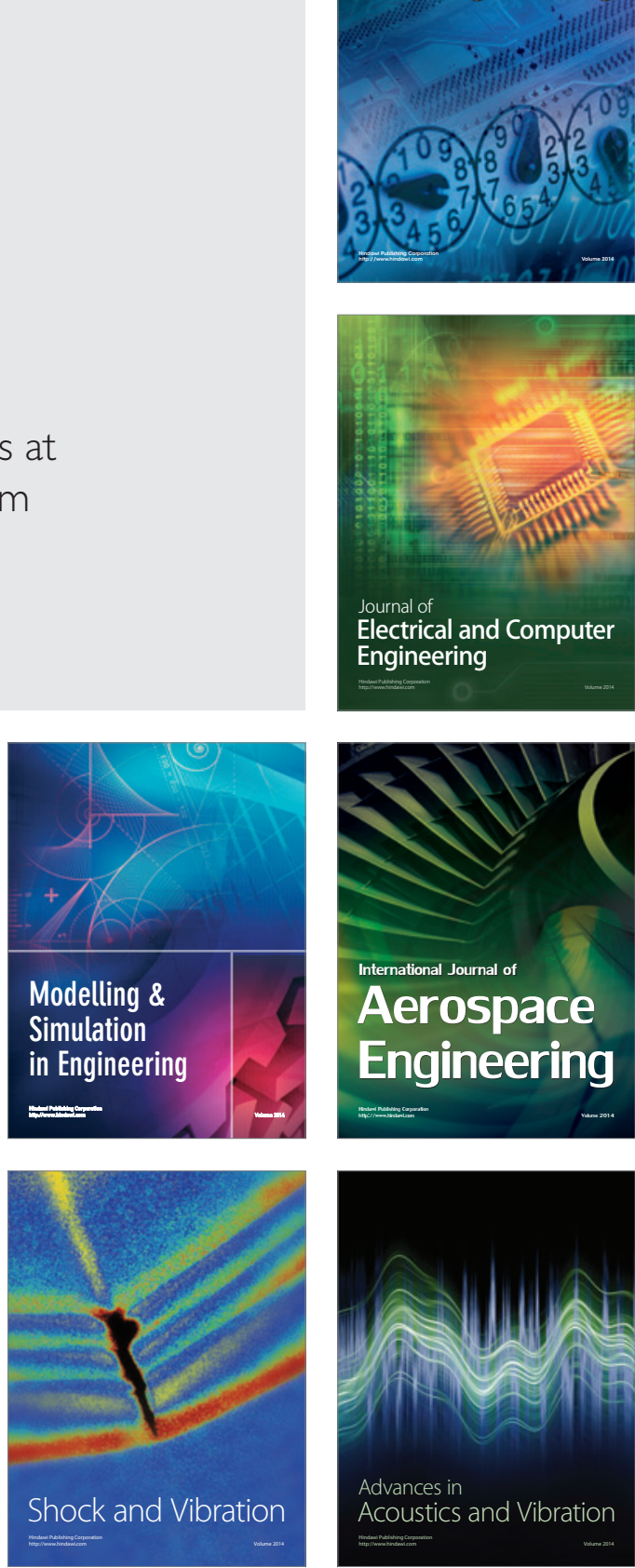